\title{
Mechanical Characterization of Carbon Nanotube- Reinforced Polymer Nanocomposite by Nanoindentation Using Finite Element Method
}

\author{
Minh-Tai Le and Shyh-Chour Huang* \\ Department of Mechanical Engineering, National Kaohsiung University of Applied Sciences, \\ Kaohsiung 80778, Taiwan, R.O.C.
}

(Received July 2, 2014; accepted February 9, 2015)

Key words: nanocomposites, nanoindentation, single-walled carbon nanotubes (SWCNTs), epoxy, finite element method (FEM)

The mechanical characterization of new generations of materials such as polymer/ carbon nanotube nanocomposites has always been challenging. In this research, the hardness and elastic modulus of single-walled carbon nanotube (SWCNT)-reinforced epoxy composites that have nonlinear elastic response were investigated using finite element analysis. A series of two-dimensional nanoindentation simulations were carried out to evaluate elastic modulus and hardness. The effects of volume percentages of the SWCNTs on the mechanical properties were evaluated. In addition, the effects of indenter geometry as well as friction coefficient on the response of nanoindentation were examined. The results indicated that the nanoindentation finite element models were able to simulate the loading-unloading responses of the epoxy/SWCNT nanocomposites. Nanoindentation investigations showed enhanced hardness and Young's modulus of nanocomposites by increasing the filler of SWCNTs as compared with pure epoxy. Future fabricated samples are expected to be promising materials for sensor and aircraft components. Furthermore, these simulated results show good agreement with experimental data from previous studies.

\section{Introduction}

Polymer/carbon nanotube composites are promising novel materials exploiting the outstanding and superior properties of carbon nanotubes in engineering materials with high mechanical and electrical properties. Nanoindentation testing is an effective and widely used technique for the characterization of the mechanical properties of these materials.

Several studies have focused on simulations of nanoindentation using a finite element method (FEM). For instance, numerical indentation studies were conducted to obtain elastic moduli, hardness values, and rare properties of plastic materials. ${ }^{(1)}$

${ }^{*}$ Corresponding author: e-mail: shuang@cc.kuas.edu.tw 
Other studies have reported experimental works on the nanoindentation of nylon 11/ clay nanocomposites ${ }^{(2)}$ and polycarbonate/multiwalled carbon nanotubes (MWCNTs) in order to understand their properties. ${ }^{(3)}$ In recent years, studies on epoxy/carbon nanotube nanocomposites have attracted major research and commercial interest. ${ }^{(4)}$ Some studies have focused on simulations and experiments exploring the effect of nanoindentation on composites and bulk materials, but to date, there have been no simulations of the nanoindentation process on SWCNT-reinforced epoxy nanocomposites.

The aim of this study is to characterize the mechanical properties of epoxy/singlewalled carbon nanotubes under nanoindentation using the numerical simulation method. A three-dimensional representative volume element (RVE) in the tensile test was simulated, and the stress-strain curve of the nanocomposite was obtained. Next, using this curve, the nanocomposite was subjected to the nanoindentation process in order to evaluate its elastic modulus and hardness as an isotropic material. Finally, the relative effects of indenter geometry, friction coefficient, and the volume percentages of the SWCNTs on the response of the elastic modulus and hardness were investigated. The novelty of the current study is the reinforced epoxy materials considered as novel materials for application in the sensor and aircraft industries.

\section{Constitutive Formulation}

The finite element model consists of a rigid spherical indenter and an elastoplastic substrate. The mechanical response of the substrate is dictated by the classical, rateindependent, isotropic elastoplasticity theory. ${ }^{(5)}$

The plastic strain rate is determined by consistency with the yield condition:

$$
\bar{\sigma}=\sigma_{\mathrm{y}}\left(e^{\mathrm{p}}\right),
$$

where $\bar{\sigma}$ is the effective stress; the yield stress $\sigma_{\mathrm{y}}$ is chosen here as a simple monotonic function of the cumulative effective plastic strain

$$
e^{\mathrm{p}}=\int \dot{e}^{\mathrm{p}} d t
$$

in which $e^{\mathrm{p}}$ is the scalar effective plastic strain rate, and the direction of flow is coaxial with the deviatoric stress.

Elastic modulus and hardness values were interpreted from the simulated data based on the composite response of the indenter and the material using the procedure devised by Oliver and Pharr. ${ }^{(6)}$

The loading part of the curve was a combination of elastic and plastic deformations, while the unloading curve was mainly dominated by elastic deformation, as shown in Fig. 1.

Fitting the unloading portion:

$$
P=\alpha\left(h-h_{\mathrm{f}}\right)^{m} .
$$

Here, $h_{\mathrm{f}}$ is the final depth after complete unloading; $\alpha$ and $m$ are fitting parameters determined by regression analysis of the initial stage of the unloading process; $P$ and $h$ were taken from the unloading curve. 


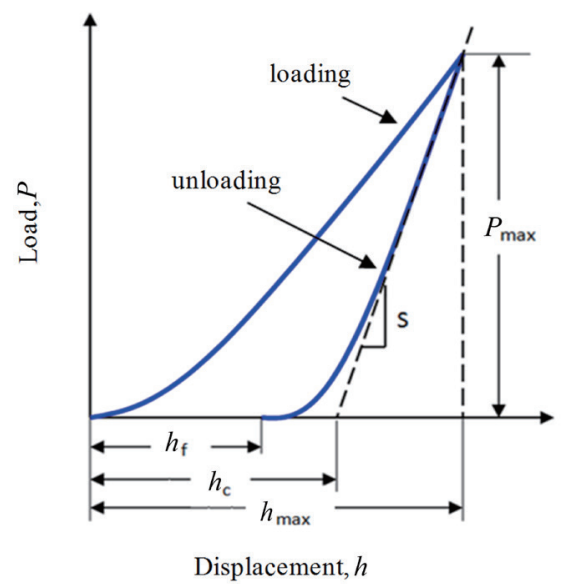

Fig. 1. (Color online) Load-displacement curve and parameters.

The slope of the unloading curve (contact stiffness) at the maximum indentation depth is given by

$$
S=\left.\frac{d P}{d h}\right|_{h=h_{\max }}=\alpha m\left(h_{\max }-h_{\mathrm{f}}\right)^{m-1} .
$$

Here, $h_{\max }$ is the maximum depth.

The contact depth of the spherical indentation $h_{\mathrm{c}}$ is shown as

$$
h_{\mathrm{c}}=h_{\max }-0.75 \frac{P_{\max }}{S} .
$$

Here, $P_{\max }$ is the maximum load at $h_{\max }$.

The contact area $A_{\mathrm{c}}$ is determined from $h_{\mathrm{c}}$ and the radius of the indenter, $R$.

$$
A_{\mathrm{c}}=\pi\left(2 R h_{\mathrm{c}}-h_{\mathrm{c}}^{2}\right)
$$

The reduced modulus is shown as

$$
E_{\mathrm{r}}=\frac{\sqrt{\pi}}{2 \beta} \frac{S}{\sqrt{A_{\mathrm{c}}}}
$$

Here, $\beta=1$ for the spherical indenter.

The indentation modulus from the Oliver-Pharr method is given by

$$
E_{\mathrm{OP}}=\frac{1-v_{\mathrm{s}}^{2}}{\left(1 / E_{\mathrm{r}}\right)-\left[\left(1-v_{\mathrm{i}}^{2}\right) / E_{\mathrm{i}}\right]} .
$$


Here, $v_{\mathrm{s}}$ is Poisson's ratio of the specimen; $E_{\mathrm{i}}$ and $v_{\mathrm{i}}$ are the elastic modulus and Poisson's ratio of the indenter, respectively.

If $E_{\mathrm{i}} \gg E_{\mathrm{s}}$, then the indenter is a rigid body.

$$
E_{\mathrm{OP}}=\left(1-v_{\mathrm{s}}^{2}\right) E_{\mathrm{r}}
$$

The nanoindentation hardness is measured as

$$
H=\frac{P_{\max }}{A_{\mathrm{c}}} .
$$

\section{Finite Element Modeling}

A finite element micromechanics model (Fig. 2) of a single group of SWCNTs embedded in a polymer matrix was developed. The model assumed three linear elastic isotropic material phases: a polymer matrix phase, a SWCNT inclusion, and an interphase connecting the matrix and inclusion. In addition, it was also assumed that the SWCNTs were solid and perfectly bonded to the interphase. A square representative volume element (RVE) with length $L=100 \mathrm{~nm}$ and square base $a=11 \mathrm{~nm}$ was used for the matrix, and the CNTs had a length $l=100 \mathrm{~nm}$ and a radius $r=5 \mathrm{~nm}$.

The SWCNT elastic modulus is 1,000 GPa, and the matrix and SWCNT Poisson's ratios are 0.4 and 0.3 , respectively. The aspect ratio of particles is assumed to be 100 . In this model, it is assumed that both the matrix and the SWCNTs are isotropic, and that the SWCNTs are perfectly bonded to the matrix. The model is subjected to plane stress. The elastic constants $E$ and $v$ and the effective deviatoric yield stress $\bar{\sigma}=\sigma_{\mathrm{y}}\left(e^{\mathrm{p}}\right)$ for this material are given in Fig. 3.

To shorten the computational time of the nonlinear solution of the nanoindentation test, a three-dimensional model is avoided. Therefore, a two-dimensional finite element model is presented. After embedding, the model is a single entity, but the elements forming the structure of the nanocomposite have properties different from those of the matrix. A typical mesh, consisting of a four-node axial symmetric element (CAX4) with 5626 elements is shown in Fig. 4.

The mesh density is highest in the vicinity of the contact region beneath the tip of the indenter and becomes increasingly coarser as the distance from the tip increases. The convergence criteria are obtained in order to ensure stable and convergent simulation results.

The friction coefficient between the tip and the specimen surface is assumed to be zero. The geometrical modeling boundary conditions are shown in Fig. 5, where the nodes on the revolution $y$-axis can move only along this axis, while all the nodes at the bottom of the sample are fixed in the $y$-direction and move in the radial direction.

Two consecutive stages, loading and unloading, were used to simulate the entire nanoindentation process. Before this procedure, an approach stage of the indenter to the surface of the nanocomposite specimen was begun, followed by the loading stage. In the loading stage, the rigid spherical indenter moved along the $y$-axis and penetrated into the sample to a maximum depth; in the unloading stage, the indenter returned to its initial position. 


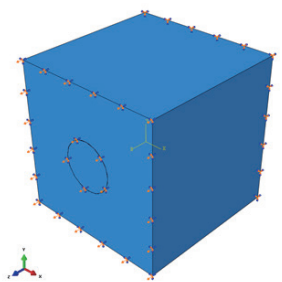

(a)

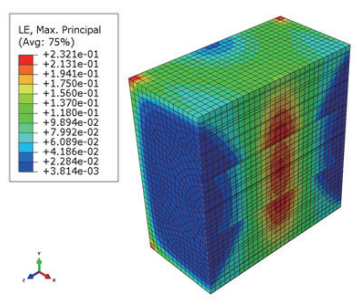

(c)

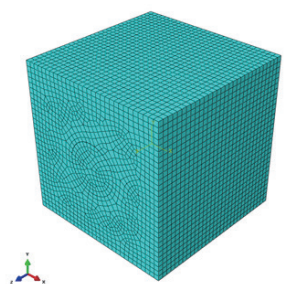

(b)

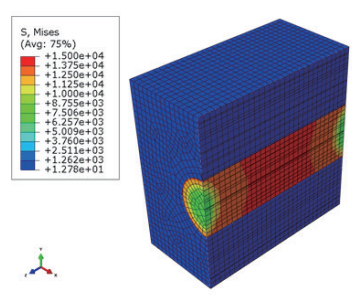

(d)

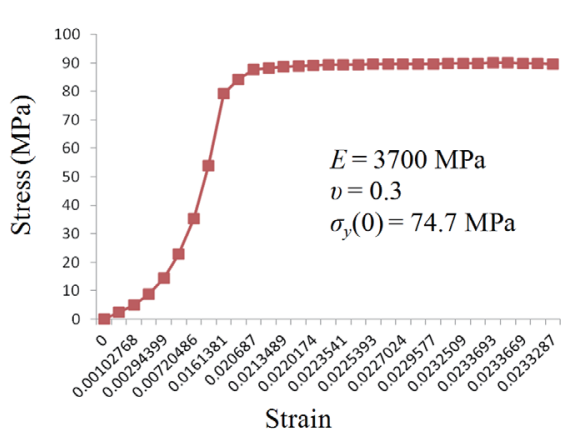

Strain

Fig. 2 (left). (Color Online) Finite element model for tensile test. (a) Boundary conditions for tensile simulation, (b) meshing for tensile model, (c) strain response of tensile model, and (d) stress response of tensile model.

Fig. 3 (right). (Color online) Stress-strain curve of epoxy/SWCNT nanocomposite.
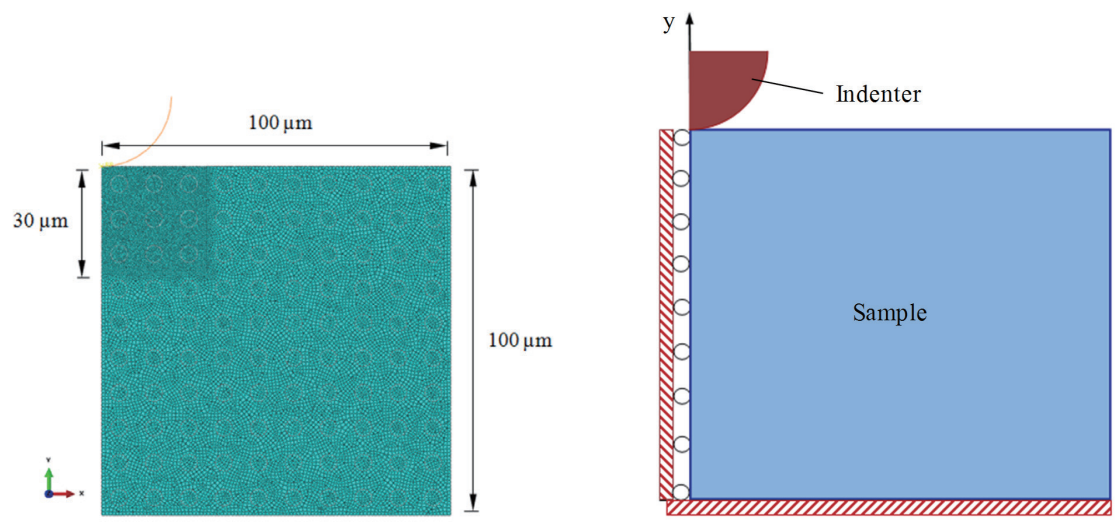

Fig. 4 (left). (Color online) Mesh of indentation model.

Fig. 5 (right). (Color online) Boundary conditions for nanoindentation model.

Figure 6 shows the total force acting on the rigid indenter in the y-direction versus the total displacement of the indenter in the $y$-direction. Note that such force versus displacement data is the key result acquired from these simulations, as it is used extensively later to calculate and evaluate the elastic modulus and hardness of the nanocomposite. Figure 7 shows a comparison of the simulation results in this study with those reported in the previous literature. (4) The following data and material properties 

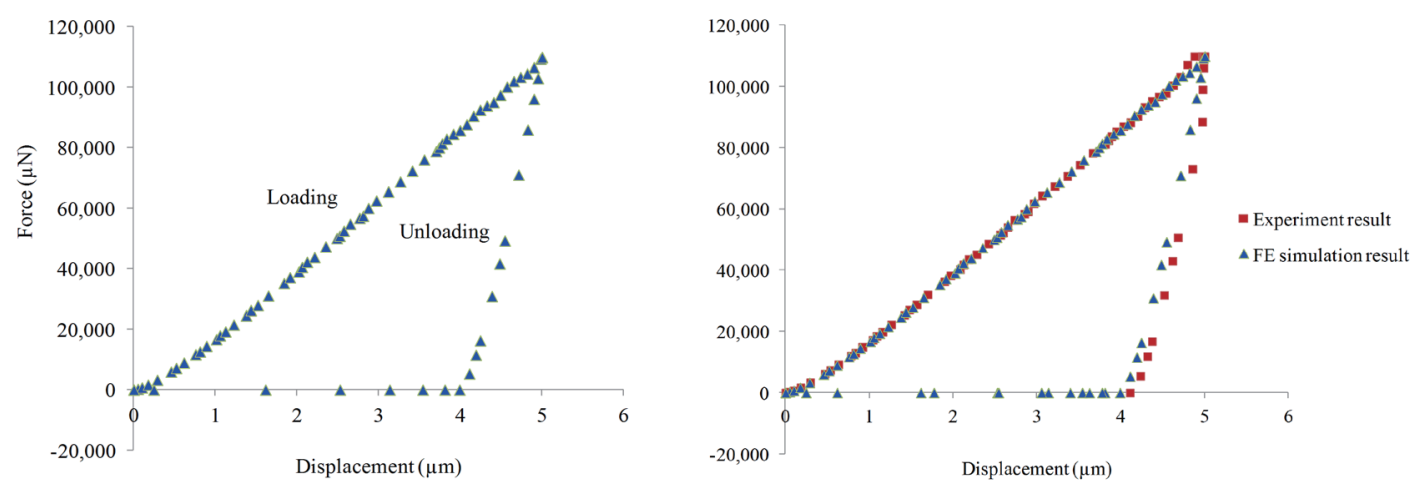

Fig. 6 (left). (Color online) Load-displacement response.

Fig. 7 (right). (Color online) Comparison between FE simulation and experiment results.

for nanoindentation analysis were obtained: Young's modulus $E$ was $3.49 \pm 0.21 \mathrm{GPa}$, Poisson's ratio $v$ was 0.3 , the initial yield stress was $74.7 \pm 3.2$, and the indentation depth was $5,000 \mathrm{~nm}$. There is a close match between the two sets of results, validating the accuracy of this study.

\section{Results and Discussion}

Figure 8 shows the equivalent stress in the sample when the indenter penetrates to a depth of 5,000 nm, and Fig. 9 shows the stress couture in the unloading stage. The Mises stress field distribution was calculated in order to obtain the condition of the elastic zone progressing to the plastic zone. It is important to mention that the mesh size near the tip of the indenter has a significant effect on the simulation results, so it should be processed as finely as possible.

The results obtained with frictionless contact and contact with friction coefficients of 0.2 and 0.4 are shown in Fig. 10, implying that the assumption of frictionless contact is acceptable. The load-displacement curves with different indenter radii is shown in Fig. 11. As shown, the required load corresponding to the indentation depth increases as the indenter radius decreases.

It can be seen that for all depths, the average modulus increased with a large percentage (1\%) of SWCNTs as compared with pure epoxy (Fig. 12). For higher volume percentages of nanotubes $(0.5$ and $1.0 \%)$, a modest increase in indentation modulus was observed, which was not consistent with the average modulus obtained from tensile test data (Table 1).

An important trend observed during indentation testing is that as the indentation depth increased, the average modulus values consistently decreased in all cases. A consistent and notable (but modest) increase in hardness was observed with the addition of nanotubes. Hardness also decreased with increasing indentation depth (Fig. 13). 

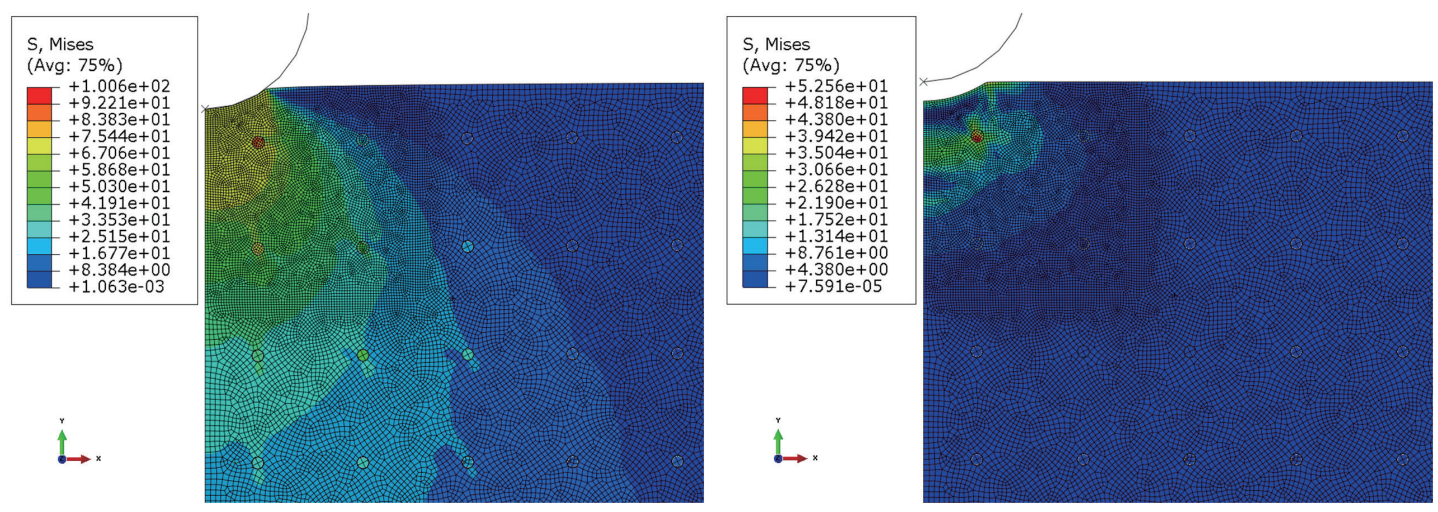

Fig. 8 (left). (Color online) von-Mises stress at depth of $5000 \mathrm{~nm}$.

Fig. 9 (right). (Color online) von-Mises stress during unloading.
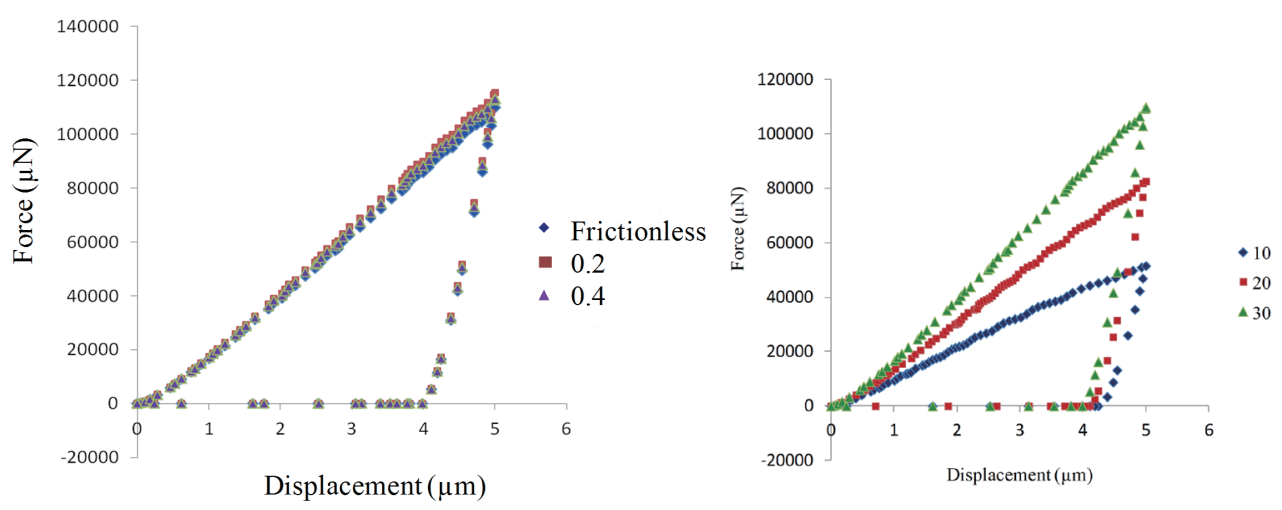

Fig. 10 (left). (Color online) Effect of friction coefficient.

Fig. 11 (right). (Color online) Effect of indenter radius.

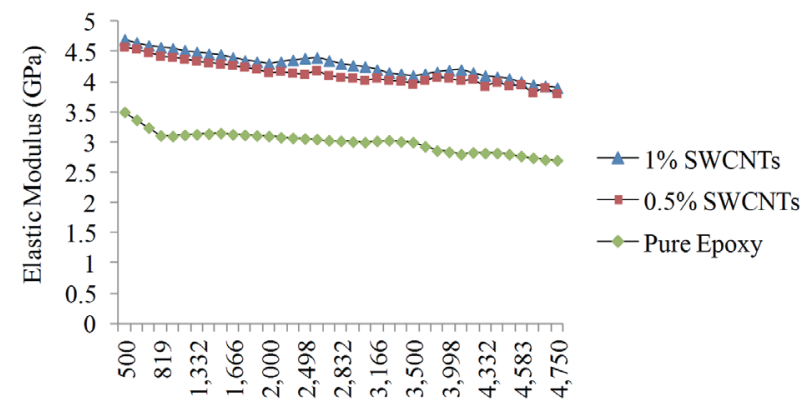

Displacement (nm)

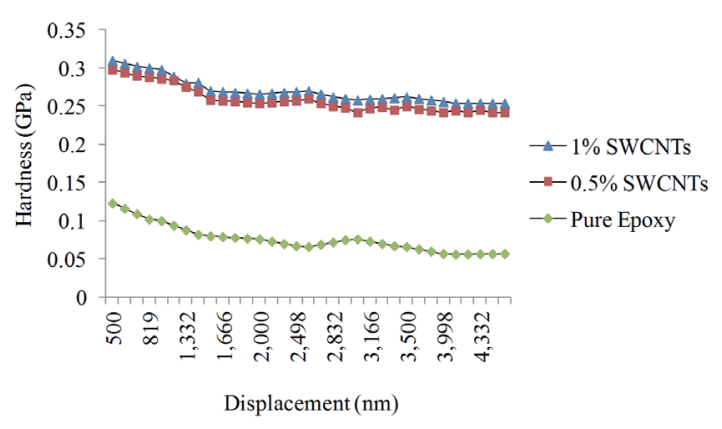

Fig. 12 (left). (Color online) Variation in elastic modulus with depth of indentation.

Fig. 13 (right). (Color online) Variation in hardness with depth of indentation. 
Table 1

Tensile-test simulation data.

\begin{tabular}{cc}
\hline SWCNT $(\%)$ & Modulus (GPa) \\
\hline 0 & 5.46 \\
0.5 & 5.96 \\
1 & 6.01 \\
\hline
\end{tabular}

\section{Conclusions}

In this study, finite element analysis and numerical simulation were successfully carried out to investigate the nanoindentation process of an epoxy/SWCNT nanocomposite. The results indicated that the nanoindentaion FE models were able to simulate the indentation loading-unloading curves of the epoxy/SWCNT nanocomposites. In addition, the results showed that the required load for indentation increased as the indenter radius decreased. The assumption of frictionless contact was also shown to be acceptable. Nanoindentation investigations showed that increasing the filler of SWCNTs, as compared with pure epoxy, enhanced both the hardness and Young's modulus of the nanocomposite. In this study, we also investigated an improved material epoxy that has not been studied previously. It is hoped that the high-strength material epoxy will be suitable for use in sensor and aircraft components. A comparison of these simulated results with those reported in other studies showed a close match between the two sets of results, validating the accuracy of the present research, and demonstrated that the continuum-based FE approach can be used to determine the loaddisplacement response of a nanoindentation test.

\section{Acknowledgements}

The authors acknowledge and thank the National Science Council of the Republic of China for their partial financial support of this study under Contract Number NSC 1022221-E-151-022.

\section{References}

1 M. Dao, N. Chollacoop, K. J. Van Vliet, T. A. Venkatesh and S. Suresh: Acta Mater. 49 (2001) 3899.

2 Y. Hu, L. Shen, H. Yang, M. Wang, T. Liu, T. Liang and J. Zhang: Polym. Test. 25 (2006) 492.

3 P. M. Nagy, D. Aranyi, P. Horváth, P. Pötschke, S. Pegel and E. Kálmán: Internet Electron. J. Mol. Des. 5 (2006) 135.

4 A. K. Dutta and D. Penumadu: J. Mater. Res. 19 (2004) 158.

5 R. Hill: The Mathematical Theory of Plasticity (Oxford University Press, New York, 1998) p. 355.

6 W. C. Oliver and G. M. Pharr: J. Mater. Res. 19 (2004) 3. 